\title{
Editorial introduction to the special issue “The Renaissance of Einstein's Theory of Gravitation"
}

\author{
Alexander Blum ${ }^{1}$, Domenico Giulini ${ }^{2}$, Roberto Lalli ${ }^{1, \text { a }}$, and Jürgen Renn ${ }^{1}$ \\ 1 Max Planck Institute for the History of Science, Berlin, Germany \\ 2 Institute for Theoretical Physics, Leibniz University of Hannover, Hannover, Germany
}

Received 18 April 2017 / Received in final form 4 May 2017

Published online 9 June 2017

(C) The Author(s) 2017. This article is published with open access at Springerlink.com

On November 25th 1915, Albert Einstein submitted to the Royal Prussian Academy of Sciences the last of a series of papers that contained the final and fundamental equation of his theory of gravitation, which he called General Relativity (Einstein 1915, 1916). This equation contains the field-theoretic law according to which the energy-momentum distribution of matter sources acts on and reacts to the gravitational field. It was the final achievement of an "intellectual odyssey," which lasted more than eight years (Renn 2007; Gutfreund and Renn 2015).

The present issue is dedicated to the centenary anniversary of this momentous scientific achievement through a series of contributions that investigate the historical trajectory of Einstein's theory of gravitation. In spite of the celebratory character of the issue, we decided not to focus on the early history of General Relativity. Einstein's own path toward the theory, its reception in different national scientific communities and the further progress until the early 1950s have been discussed in an enormous amount of scholarly work during the past decades. Instead, we prefer to take this opportunity to explore in more detail the post-World War II developments of the theory, which only recently has become the subject of a lively debate among historians of science and physicists actively working on General Relativity and closely related fields.

This issue of EPJH aims to give new insights into the historical process through which Einstein's theory of gravitation came to turn into that fruitful and exciting branch of the physical research we know today. This process looked so splendid to some of the protagonists that physicist Will $(1986,1989)$ dubbed it the "Renaissance of General Relativity". But what is meant exactly by Renaissance? What kind of complex process does the term try to describe? Was it a mere consequence of the general growth of physics in the post-WW II period? Or did the phenomenon entail deeper epistemic transformations?

a e-mail: rlalli@mpiwg-berlin.mpg.de 
From a superficial perspective, the history of Einstein's theory of gravitation might look like an inevitable success story. The recent detection of gravitational waves (Abbott et al. 2016), predicted by Einstein a century ago, have once more impressively underlined the central role that General Relativity will play in our understanding of fundamental interactions and cosmology. Today every physics student is told that General Relativity is one of the pillars of modern physics, together with quantum mechanics and quantum field theory.

But this has not always been the case. After an initial burst of excitement following the 1919 announcement that one of the few predictions of Einstein's theory-the gravitational deflection of light rays-had been confirmed, the theory underwent a period of stagnation, which lasted from the mid-1920s to the mid-1950s. Historian of physics Eisenstaedt $(1986,1989)$, who was the first to study the long-term history of General Relativity, called this phase the "low-water-mark" period. During this period only a few scientists worked on a theory that was seen by the majority of the physics community as mathematically extremely expensive with very little physical yield. And even physicists with undeniable strong mathematical inclination, like Pascual Jordan, were initially appalled by the "mismatch between the simplicity of the physical and epistemological foundations and the annoying complexity of the corresponding thicket of formulae" (Jordan 1952, p. 5).

Most of the invested work was seen to yield only either formal improvements or minor corrections to Newtonian predictions. As a result, the majority of theoretical physicists around the mid-1920s lost interest in the theory and preferred to focus on the far more exciting development of quantum mechanics, its plethora of applications to micro- and solid-state physics, which gave rise to much stronger and more fruitful connections with experimental activities and, last but not least, also promised much better career prospects. So, for a long time, a neo-Newtonian interpretation of General Relativity prevailed as the dominant attitude, where General Relativity was viewed merely as providing small corrections to Newtonian gravity, neglecting its fundamental aspects like the unification of the inertial and gravitational fields altogether.

An example of this attitude is that the physical meaning and domain of applicability of the full exterior Schwarzschild solution (including the horizon) remained unclear until the 1960s. There was a great amount of confusion as to whether the event horizon contained in this solution corresponded to a real spacetime singularity or whether its apparently singular nature was merely an artifact of an unsuitable choice of coordinates (Eisenstaedt 1987). This is not to say that during the low-water-mark period there was no important work on the Schwarzschild solution. Eisenstaedt himself and Luisa Bonolis in this volume show that researchers made significant progress and offered a number of insights on this issue, in some cases with direct connections to physical applications. The most important was certainly the work of Robert Oppenheimer and his co-authors on the application of General Relativity to stellar collapse in 1939 (Oppenheimer and Snyder 1939; Oppenheimer and Volkoff 1939). Nonetheless, these important advances did not become part of the shared knowledge of the experts on General Relativity. The criteria scientists used to evaluate the significance of specific advances and to define which were the important questions to be addressed varied considerably. What in hindsight could be considered important results were often ignored, and some of them remained controversial for decades. The relevance of General Relativity for the discipline of physics as a whole was also cause of disagreement. Oppenheimer himself strongly encouraged students and younger researchers to work on topics different from General Relativity. He did so in the mid-1950s, when another authoritative theoretical nuclear physicist, Wheeler, was instead making General Relativity the main focus of his research agenda. Such a fate of grossly diverging attitudes was also suffered by the theory of gravitational radiation. Here confusion reigned even as to whether gravitational waves were physically real, e.g., in the sense 
that they can carry energy from the source to the distant observer. Quite remarkably, Einstein himself came to doubt their physical existence in the 1930s (Kennefick 2007, pp. 79-104).

By the 1970s, the status of Einstein's theory of gravitation was completely different: the theory was perceived as an important, empirically well tested branch of theoretical physics, which had also produced a brand new and successful sub-discipline: relativistic astrophysics. It is important to note that the impact of General Relativity onto astrophysics was by far not exhausted by quantitative corrections, but also, and more essentially, by its addition of new qualitative features, e.g., concerning the structure and stability of stars, the formation of Black Holes, the emission of gravitational waves, and gravitational lensing as tools for mass detection. With at least one notable exception (Goenner 2017), most historians of science and physicists agree that sometime by the end of the 1960s a significant process had occurred, which might be described as a renaissance of the theory (see also Thorne 1994; Kaiser 2000; Kragh 2002; Kennefick 2007). In addition, the intimate connection of General Relativity with various mathematical branches with no previously established close connection with physics, like non-Riemannian differential-geometry and differentialand point-set topology, ceased be perceived as mere excess baggage. Rather, it turned into a positive aspect connected with the hope that new insights will emerge at the interface between mathematics and physics, eventually to the advantage of both sides. As an example we mention the 1967 Battelle Recontres lectures in mathematics and physics (DeWitt and Wheeler 1968), which, amongst others, brought together eminent mathematicians with no previous record in relativity, like Raoul Bott, Paul Federbush, Sigurdur Helgason, Stephen Smale, and Norman Steenrod, with physicists and mathematicians who had already worked in the field of relativity, including Brandon Carter, Yvonne Choquet-Bruhat, Bryce DeWitt, Cécile DeWitt-Morette, Robert Geroch, Stephen Hawking, André Lichnerowicz, Roger Penrose, Tullio Regge, John Wheeler and James York. The belief in a fruitful interaction was expressed succinctly on the cover of the proceedings volume, the title of which ends with $M \cap P \neq \emptyset$.

The descriptions of what this renaissance was, however, vary considerably, but most of them share a specific bias concerning the historical development of scientific theories: Next to Newton's Classical Mechanics, Einstein's General Relativity is often regarded as the prototypical example of a breakthrough in scientific theory associated with a framework created by a single ingenious scientist, on which all later developments are built, filling in the details without the need to revise the foundation. Consequently, the further development of the framework can only consist in integrating novel empirical evidence, working out the implications of the fundamental equations, and the introduction of new calculational techniques. Accordingly, the framework itself has no history of its own and fundamental progress can only occur through major upheavals. This view of theory (non-)development thus matches perfectly with the common reading of Kuhn's theory of scientific revolutions as radical paradigm changes, followed by long periods of normal science consisting of puzzle-solving. The latter are usually less important when trying to locate the decisive strategic moments in the history of theory formation. In reviewing, e.g., the history of gravitational waves on the occasion the recent discovery, the one constant is Einstein's "prediction" of 1916, with some puzzles and ambiguities resolved along the way. The debates concerning the existence of gravitational waves, which went on for at least 40 years, are then, also by historians of science, generally viewed as being a mere "comedy of errors" caused by lack of empirical evidence, lack of funding, disciplinary divides, lack of communication, and even personal idiosyncrasies.

This bias now severely restricts the scope of how one can interpret the evident turning point that is usually referred to as the Renaissance of General Relativity: It might be due to the influx of new empirical evidence, made possible by novel 
technologies, it might be an almost trivial consequence of the postwar political situation, with the unprecedented flow of money into virtually every branch of physics, or it might be due to the solution of a particularly persistent puzzle, which had been a bottleneck for further progress. Within this scope, it remains, however, difficult to explain how it came to (a) a burst of theoretical advances in several unrelated areas of General Relativity, which occurred (b) years before the major discoveries of 1960s radio-astronomy (the discovery of quasars in 1963, of the Cosmic Background Radiation in 1965, and of pulsars in 1967). And even if this sudden eruption were brushed aside as a mere coincidence, the question would remain why up to this turning point, so many major figures in General Relativity persistently mistrusted the qualitatively new implications of the theory, such as the possibility of unstoppable gravitational collapse or, to return to our example, the existence of gravitational waves.

These puzzles indicate, in our view, that the entire idea of scientific progress informing this narrative is seriously deficient. It seems more plausible, instead, that theories do have history and that their history does not consist merely in puzzlesolving. Indeed, if we admit that, in the Renaissance period, the conceptual foundation of General Relativity itself underwent a development, we can explain (a) why so many persistent problems suddenly became solvable within a relatively short time period; (b) why General Relativity was transformed from a marginal theory, primarily of mere philosophical and mathematical interest, into a vibrant field of research; and (c) how relativists were able to react so quickly to the unexpected experimental breakthroughs in astrophysics. This change did not consist in a modification of the foundation laid by Einstein: the Einstein Equation remains the cornerstone of General Relativity to this day. Rather, we are looking at an extension of the foundation: The theory of 1915 was insufficient to reach firm conclusions without being complemented by intuitions drawn from the resources of pre-relativistic physics or (for the case of cosmology) by philosophical considerations that were hardly generalizable to more mundane problems. Finding a general way to extract the physical content of the theory first became a major concern in the Renaissance years, with many papers opening with remarks concerning the difficulty of interpreting General Relativity. Only after the central issues had been resolved in the Renaissance was General Relativity applicable to any given physical problem, providing an interpretation in its own terms. The Renaissance was thus not a mere agglomeration of isolated results, but a global transformation in the character of the theory. Such a global transformation, which in Kuhnian terms might be described as a "paradigm shift," was hence not the premise, but rather the result coming at the end of a long period of problem-solving within General Relativity.

While historically-minded physicists have long been interested in the establishment of general relativity by Einstein in the 1910s, we hope that the papers in this special issue will demonstrate that the Renaissane period is of similar importance to those interested in the conceptual foundations of general relativity and its historical development (see also the programmatic articles by three of the editors, Blum et al. 2015, 2016). Given the focus of this journal, the authors in this volume have addressed from different perspectives one central aspect of the process of the Renaissance of Einstein's theory of gravitation: Its establishment as a field of study in its own right within the discipline of physics, rather than as an object of mere philosophical or mathematical analysis. It is the return of General Relativity to the mainstream of physics that the authors have discussed in different and pertinent cases and using a variety of approaches, some of which are somewhat different with respect to the style of papers that usually appear in the EPJH. The order of papers is more a conceptual than a chronological one, as the authors have discussed quite different aspects that cannot be easily considered as following a purely chronological progressive development. 
The first paper in this special issue, by Alexander Blum and Thiago Hartz, focuses on the role of the program of constructing a quantum theory of gravity in the renaissance of General Relativity. They do this through a close reading and contextualization of a heretofore unpublished historical document, a report on a workshop on the quantization of the gravitational field, held in Copenhagen in the summer of 1957, published here for the first time. Held several months after the famous Chapel Hill conference, the Copenhagen workshop was arguably the first ever scientific meeting dealing solely with question of quantizing gravity and provides us with a unique glimpse at the role that this problem played in the physics of the time in general, and in the Renaissance of General Relativity in particular.

The notion of quantizing General Relativity carried with it an air of "domestication," whereby General Relativity would be brought from the realm of classical field theory (which the attempts by Einstein and others to construct a unified field theory did not transcend) into the domain of quantum theory, which formed the basis for most of the work in the physics mainstream, from solid state to high-energy nuclear physics. As the authors outline, it was Bryce DeWitt (the author of the report) who attempted to integrate these scattered attempts at domestication into the emerging renaissance community, by bringing together various approaches and finding a common agenda. As is well-known, the attempts at constructing a theory of quantum gravity have to this day not met with ultimate success, and there is still no universally accepted approach to the problem. The authors thus present DeWitt's attempt to find such a common agenda as a failed attempt to bring General Relativity back into the physics mainstream by strengthening its ties to high-energy physics and quantum field theory. This "physicalization" of General Relativity instead happened several years later solely via its connections to astrophysics and astronomy, while its relation to quantum theory remained elusive.

The theoretical discourse was in any case not the only way in which the General Relativity returned to the field of physics; there was also an exponential increase in experimental activities aimed at testing the predictions of the theory. Peebles' article in this volume reviews the early attempts to establish the field of the experimental study of gravity in the decade between the late 1950s and the late 1960s. Peebles argues that the growth was so impressive that one can well say that the field was actually born in that period. This is why he named the process the "naissance of experimental gravity physics". Building on his deep firsthand knowledge of the field, Peebles' review covers all the relevant scientific activities in experimental gravity physics of the period, although its main focus remains the pioneering work of Robert Dicke and of the group Dicke established in Princeton, where Peebles himself earned his PhD. The activity of this group, Peebles shows, had a relevant role in sparking this kind of research and strengthening its position as a relevant part of the physics endeavour. As for the historical factors underlying the process of the "naissance of experimental gravity physics," Peebles especially stresses the relevance of technological advances. New technologies were an essential component, for they allowed to draw unprecedented connections between the theory and the physical world. But by focusing on Dicke's own trajectory and his decision to change direction in mid-career, Peebles also shows that other factors, which cannot be reduced to the new possibilities opened by technological advances, played a similarly important role, such as the formulation of alternative theories of gravitation (such as the Brans-Dicke theory, also known as the Jordan-Thiry-Brans-Dicke theory) that provided both a theoretical background and the motivation to perform experiments designed to provide a crucial support to one of the competing theories, or the ability of individual experimenters to make use of the advancements in technology or in fields different from their own area of expertise. From the various different factors that shaped the early history of experimental 
gravity physics, Peebles draws some general lessons that are intended as food for thought for active experimental physicists.

Besides experiments designed to putting the theory to the test, the experimentalobservational status of the general theory of relativity changed completely in the renaissance period. The two aspects of this change are in the area of gravitational-wave research and in the field of relativistic astrophysics. To these two subjects are devoted the last three articles of the volume. The experimental activity aimed at detecting gravitational waves exploded in the 1970s and there is good evidence that this activity was mostly a consequence of Weber's 1969 announcement that his attempts in this direction had been successful (Weber 1969, 1970). Up to this moment, Weber had pursued this activity alone or in collaboration with a few students and assistants. After the announcement, he had to face a number of controversies with his peers, who started to distrust his results after about 1972/73 (Collins 2004). To this dramatic, visionary figure, who may well be argued to have not obtained the credit he deserved during the time of his career, is dedicated a personal recollection by astronomer and historian of physics Virginia Trimble, who was also Weber's wife for the final twentyeight years of his life.

The focus on the personal trajectory of a visionary and controversial scientist reminds us that the history of physics cannot simply be understood as the progressive accumulation of knowledge, but that several factors enter the development of science, some of them of non-scientific nature. And this is especially true in the case of controversies, where the debate is not only between individuals, but involves different social groups defined by disciplinary boundaries or different training. This is the perspective proposed by Daniel Kennefick in his paper "The Binary Pulsar and the Quadrupole Formula Controversy". In his book on the history of the theoretical quest on gravitational radiation, Kennefick (2007) had already shown that these theoretical developments were clouded by disagreements as to whether gravitational waves existed and which properties they had. The consensus on the fact that energycarrying gravitational waves existed was in fact achieved only during the renaissance period. Even after the majority of theoreticians working on the theory of General Relativity came to accept the physical reality of gravitational waves, several aspects continued to remain matters of debate. Among them, the most pressing were whether binary systems decay because of gravitational damping and the related question as to whether the quadrupole formula first derived by Einstein (1918) in the linearized approximation scheme was the correct way to deal with this problem. Kennefick's contribution to this volume deals with the development of this theoretical controversy and the twofold role of the discovery of the binary pulsar system PSR 1913+16 in 1974 and the subsequent observation of its decay, firstly in sharping the theoretical controversy and then in closing it. In his narrative, Kennefick focuses in particular on the epistemic aspects related to the social separation of the involved scientists in different communities: that of application-/calculation-minded physicists on one side, and that of physicists with a strong commitment to mathematical rigor on the other. Kennefick stresses the relevance of this kind of social structure for the way in which such controversies evolve.

Finally, the birth of relativistic astrophysics, the crowning element of the return of General Relativity to the mainstream of physics, is addressed in the paper by Luisa Bonolis entitled "Stellar structure and compact objects before 1940: Towards relativistic astrophysics". The author looks at this process from the perspective of the continuity of research on astrophysical compact objects that became an active branch of research since the mid-1920s, after the development of quantum mechanics and its application to solid state physics. In this issue, Bonolis presents the first part of a twopart paper on the history of the astrophysics of highly dense objects up to its transformation into relativistic astrophysics during the 1960s. In this first part presented 
here, the early phases of these research activities up to the beginning of the Second World War are investigated. According to Bonolis, only by following the long-term development of the theoretical study of the stellar structure of compact objects and the connection of this research with contemporary developments in other branches of theoretical physics is one able to properly understand what is considered as an essential element of the "physicalization" of Einstein's gravitational theory; namely, the application of the theory to solve the physical problems related to the newly discovered astrophysical objects of quasars and pulsars, which since their discoveries required the application of a non-Newtonian theory of gravitation.

While the articles in this volume shed lights on important aspects of the process to which the volume is dedicated, the phenomenon was so huge and complex that many other aspects still remain to be investigated by historians of science and physicists. For starters, the list of topics that were at the focus of the renaissance is of course by no means exhausted by the papers presented in this issue. One such important issue is the problem of motion in General Relativity: Einstein's equations imply by way of integrability conditions a kind of local conservation concerning the energy-momentum exchange between the gravitational field and matter, thereby strongly restricting, or sometimes even determining, the dynamics of the latter. These integrability conditions may jeopardize consistency of approximation schemes if not properly taken into account. First advances were made already in the late 1930s through the work of Einstein et al. (1938, see also Havas 1989) but the problem remained largely neglected until it was unignorably put back onto the agenda of theoretical astrophysicists in the mid-1970s (Ehlers et al. 1976) where it remained as an active field of research ever since (Blanchet et al. 2011; Pützfeld et al. 2015). The problem of motion for extended bodies, in the course of the renaissance and beyond, also represents a promising field for historical research; see, e.g., Dixon's contribution "The New Mechanics of Myron Mathisson and Its Subsequent Development" in Pützfeld et al. (2015).

Another central conceptual development of the renaissance is a sharpening of the notion of singularity, culminating in the singularity theorems of Roger Penrose and Stephen Hawking in the mid-1960s (Penrose 1965; Hawking and Penrose 1970). The question of singularities provides an enticing case study for general questions of theory and concept development. The notion of a singularity had been around for a long while and had served as a criterion for excluding solutions of the Einstein equations as physically irrelevant. Such arguments had been used, e.g., in the 1930s to deny the existence of plane gravitational waves, and also to discredit simplified models of gravitational collapse or big-bang cosmology. One important renaissance development was the definitive disentangling of coordinate and genuine (non removable) singularities; this was just as much a sociological advance as it was a conceptual one, given the fact that many physicists and mathematicians appear to have been clear on this matter already in the 1930s, but that it only became generally accepted once there was an established community out of which such insights could spread. Here historians have the opportunity to study how a research field develops a memory and thus why, after the renaissance, John Stachel's dictum that everything worth discovering in General Relativity was discovered at least twice might no longer hold (Stachel 1992).

But going beyond this disambiguation, there was also the problem of finding a positive characterization of a proper (non coordinate) singularity that also made some intuitive physical sense. The community would ultimately agree on a definition as used by Penrose, Hawking and Geroch based on the notion of geodesic incompleteness, but this was still a compromise lacking many desirable features (Geroch 1968). Here the task for future historians could be to trace and understand its origins and the reasons for (and the conceptual impact of) its ultimate general acceptance, despite its flaws. This raises further more general questions on how the emerging community agreed upon matters such as formal definitions or standards of argument and mathematical 
rigour, and what impact formal-mathematical work had, e.g., on the inquiries into actual physical black holes.

Similarly probing epistemological questions may well be asked also concerning those topics that are addressed in the current volume. For the history of science progresses not just by broadening its scope, but also by delving ever deeper into the dynamics of the evolution of science. Detailed reviews, often written by physicists, such as Jim Peebles' contribution to this volume or Malcolm Longair's magisterial book The Cosmic Century on the history of astrophysics, often form the starting point for the kind of historico-critical analysis that historians of science (at least those with a strong interest in the actual content of the science under study) aspire to. Questions such as the ones we formulated for the case of singularities are still unanswered also for the case of one of the central topics of this issue, gravitational waves: How did formal-mathematical existence proofs for wave solutions of the Einstein equations (initiated by Felix Pirani and Andrzej Trautman in the late 1950s) actually relate to the more physical questions of gravitational wave emission and absorption discussed in this issue?

This question finally brings us to the third dimension in which the future historiography of General Relativity should and will progress: Besides the thematic broadening and the epistemological deepening, we also have simple chronological progression. The renaissance for all its import is certainly not the end of the exciting history of the singular theory that is General Relativity. All of the stories told and questions raised and answered in this issue can be extended towards the present. We have already outlined for the case of gravitational waves the challenge of pursuing and understanding the development that led from total skepticism about gravitational waves in the mid-1950s to their ultimate discovery 60 years later. Similarly, we see in the article by Blum and Hartz that in 1957 there was still considerable optimism that the construction of a quantum theory of gravity would be achieved within the next few years. When and for which reasons did this change and how did the different approaches to quantum gravity interact with and affect the General Relativity community at large in the following decades? In formulating these questions one can already see that we are here entering a territory that historians of science tend to eschew: Dealing with the history of scientific research programs that have not yet reached an accepted definite conclusion.

One argument often brought forth is that historians should not meddle in scientific matters that are still unresolved. This is hardly a tenable position: Political historians are very much engaging with the history of the 1970s, say, dealing with social and geopolitical issues that are by any standard unresolved to this day. And even if one would want to argue that history of science is somehow different in this regard, science can hardly be so neatly compartmentalized into solved and unsolved problems. Attempting to do so would lead to a highly incomplete picture of the scientific development in the second half of the twentieth century, or to an ultimately superficial understanding of merely the institutional and social aspects of modern science. The renaissance of General Relativity is, in fact, a perfect illustration of the fact that these institutional and social developments cannot be understood in isolation, but only through their intimate interaction with the content of science. For this reason, we conclude this introduction by stressing that there are plenty of open physical questions that the renaissance generation handed down to us in the hope that they may pique the interest of young physicists and historians of science alike.

Amongst the open issues is, of course, the overarching problem of how to reconcile the theoretical description of gravity with that of the remaining fundamental interaction. In absence of obvious phenomenological data the physical need for such a unification is usually seen in genuine predictions of General Relativity, like that of singularities and other spacetime features considered to be pathological. Currently 
it is rather unclear of whether this unification process will be more like a "quantization of gravity" or rather a "gravitization of Quantum Theory" (Penrose 2014a,b) or, perhaps most likely, both at the same time. We know that the impact of relaxing the spacetime symmetries of Special Relativity (Poincaré group) onto our familiar concepts of Quantum-Field Theory is dramatic: no particles, no vacuum, no scattering theory! There is so far no well founded intuition regarding proper replacements that might work beyond semi-classical situations. And even in semi-classical contexts the coupling of the classical gravitational field (the metric) to ordinary quantum mechanical matter is not understood in detail and from first principles. How does an atom react to the non-newtonian components of the Einsteinian gravitational field (i.e. gravitomagnetism and gravitational waves) and how does a non-classical delocalized state of a quantum system source a gravitational field? How do we formulate the equivalence principle in a way that applies to quantum matter (no point particles, no world lines, no clocks)? These are apparently mundane questions which have so far not received accepted answers.

Sill further down, on a purely classical level, hard technical problems remain. The full (non-linear) stability of even the simplest non-trivial solutions, like that of Schwarzschild and Kerr, are unknown. Sound approximation schemes in cosmology are lacking, which means that we do not know how to properly derive the Friedmann equations (which underlie our cosmological standard model) as controlled approximation to the full Einstein equations in situations with only approximate homogeneity. Calculations of gravitational radiation-reaction upon the sources are much more difficult than in the linear case of electrodynamics and certainly plagued with the same pathologies (runaway solutions). And even without radiation reaction, the analytical treatment of the equations of motion of structured (and necessarily spatially extended) bodies in strong gravitational fields is still not in a satisfying form, free of mathematical tricks and uncontrolled approximations (Blanchet et al. 2011; Pützfeld et al. 2015).

This list could easily be continued. But the fact that hard theoretical problems remain unsolved should not mislead the reader into thinking that this theory - General Relativity - lacks precision and foundation. Quite the opposite: The bigger the success the more ambitious our criteria for proper understanding become and develop. Modern precision tests in astrophysics and cosmology show that General Relativity can clearly bear comparison with the best predictive theories in all of physics. Moreover, the mathematical formulation of its physical and epistemological foundations is certainly no less adequate, and presumably even better, than that of any other fundamental theory in physics. We therefore feel entitled to predict that its future will be as astonishing and revealing as its past has already been.

\section{References}

Abbott, B.P. et al. 2016. Observation of Gravitational Waves from a Binary Black Hole Merger. Phys. Rev. Lett. 116: 061102-1-061102-14

Blanchet, L., A. Spallici and B. Whiting. Eds. 2011. Mass and Motion in General Relativity. Springer, Dordrecht

Blum, A., R. Lalli and J. Renn. 2015. The Reinvention of General Relativity: A Historiographical Framework for Assessing One Hundred Years of Curved Space-time. Isis 106: 598-620

Blum, A., R. Lalli and J. Renn. 2016. The Renaissance of General Relativity: How and Why It Happened. Annalen der Physik 528: 344-349

Collins, H. 2004. Gravity's Shadow. Univ. of Chicago Press, Chicago

DeWitt, C. and J.A. Wheeler. Eds. 1968. Battelle Recontres - 1967 Lectures in Mathematics and Physics $-M \cap P \neq \emptyset$, W.A. Benjamin, Inc., New York 
Ehlers, J., A. Rosenblum, J. Goldberg and P. Havas. 1976. Comments on gravitational radiation damping and energy loss in binary systems. The Astrophysical Journal 208: L77-L81

Einstein, A. 1915. Feldgleichungen der Gravitation. Preuss. Akad. Wiss. Berlin, Sitzungsberichte der Physikalisch-mathematischen Klasse: 844-847

Einstein, A. 1916. Die Grundlage der Allgemeinen Relativitätstheorie. Annalen der Physik 354: $769-822$

Einstein, A. 1918. Über Gravitationswellen. Preuss. Akad. Wiss. Berlin, Sitzungsberichte der Physikalisch-mathematischen Klasse: 154-167

Einstein, A., L. Infeld and B. Hoffmann. 1938. The gravitational equations and the problem of motion. Ann. Math. 59: 65-100

Eisenstaedt, J. 1986. La Relativité Générale à l'Étiage: 1925-1955. Archive for History of Exact Sciences 35: 115-185

Eisenstaedt, J. 1987. Trajectoires et Impasses de la Solution de Schwarzschild. Archive for History of Exact Sciences 37: 275-357

Eisenstaedt, J. 1989. The Low Water Mark of General Relativity. In Einstein and the History of General Relativity, Einstein Studies, Vol. 1. Ed. Don Howard and John Stachel. Birkhäuser, Boston, pp. 277-292

Geroch, R. 1968. What is a Singularity in General Relativity? Annals of Physics 48: 526-540

Goenner, H. 2017. A golden age of general relativity? Some remarks on the history of general relativity. General Relativity and Gravitation 49: 42. doi:10.1007/s10714-017-2203-1

Gutfreund, H. and J. Renn. 2015. The Road to Relativity. Princeton Univ. Press, Princeton, NJ

Havas, P. 1989. The Early History of the "Problem of Motion" in General Relativity. In Einstein and the History of General Relativity, Einstein Studies, Vol. 1. Ed. Don Howard and John Stachel. Birkhäuser, Boston, pp. 234-276

Hawking, S.W. and R. Penrose. 1970. The singularities of gravitational collapse and cosmology. Proceedings of the Royal Society London Series A 314: 529-548

Jordan, P. 1952. Schwerkraft und Weltall, Vieweg \& Sohn, Braunschweig

Kaiser, D. 2000. Roger Babson and the Rediscovery of General Relativity. In Making Theory: Producing Theory and Theorists in Postwar America. Ph. D. dissertation. Harvard Univ. Press, Cambridge, MA, pp. 567-595

Kennefick, D. 2007. Traveling with the speed of thought: Einstein and the quest for gravitational waves. Princeton Univ. Press, Princeton, NJ

Kragh, H. 2002. Quantum generations: A history of physics in the twentieth century. Princeton Univ. Press, Princeton, NJ

Oppenheimer, J.R. and H. Snyder. 1939. On continued gravitational contraction. Physical Review 56: 455-459

Oppenheimer, J.R. and G.M. Volkoff. 1939. On massive neutron cores. Physical Review 55: $374-381$

Penrose, R. 1965. Gravitational collapse and space-time singularities. Physical Review Letters 14: $57-59$

Penrose, R. 2014a. On the Gravitization of Quantum Mechanics 1: Quantum State Reduction. Foundations of Physics 44: 557-575

Penrose, R. 2014b. On the Gravitization of Quantum Mechanics 2: Conformal Cyclic Cosmology. Foundations of Physics 44: 873-890

Pützfeld, D., C. Lämmerzahl and B. Schutz. Eds. 2015. Equations of Motion in Relativistic Gravity. Springer, Dordrecht

Renn, R. Ed. 2007. The Genesis of General Relativity. Springer, Dordrecht

Stachel, J. 1992. The Cauchy problem in general relativity-the early years. In Studies in the History of General Relativity, Einstein Studies, Vol. 3, edited by J. Eisenstaedt and A.J. Kox. Birkhäuser, Boston, pp. 407-418

Thorne, K. 1994. Black Holes and Time Warps: Einstein's Outrageous Legacy. Norton, New York

Weber, J. 1969. Evidence for the discovery of gravitational radiation. Phys. Rev. Lett. 22: $1320-1324$ 
Weber, J. 1970. Gravitational radiation experiments. Phys. Rev. Lett. 24: 276-279

Will, C.M. 1986. Was Einstein Right? Putting General Relativity to the Test. Basic Books, New York

Will, C.M. 1989. The Renaissance of General Relativity. In The New Physics. Ed. Paul Davies. Cambridge University Press, Cambridge, pp. 7-33

Open Access This is an open access article distributed under the terms of the Creative Commons Attribution License (http://creativecommons.org/licenses/by/4.0), which permits unrestricted use, distribution, and reproduction in any medium, provided the original work is properly cited. 